\title{
Determining the level of structural survivability to control the reliability of innovative economic systems
}

\author{
Anna Ponikarova ${ }^{1, *}$, Maksim Zotov ${ }^{1}$,and Irina Ponikarova ${ }^{2}$ \\ ${ }^{1}$ Department of Management and Entrepreneurship, Kazan National Research Technological \\ University, 420015, 68Karl Marx Street, Kazan, Russia \\ ${ }^{2}$ Department of Engineering, Computer graphics and Computer-aided design, Kazan National \\ Research Technological University, 420015, 68Karl Marx Street, Kazan, Russia
}

\begin{abstract}
Currently, the development of modern economic systems takes place in a continuous increase in the requirements for the quality and reliability of their innovative functions. This creates the need to search for new sources of their increase in the course of innovative development of companies. In this paper, the study of existing approaches to assessing the reliability of the development of innovative economic systems based on determining the level of their structural survivability. It is noted that the achievement of reliability of innovative development of the organization is provided by choosing the most effective project, taking into account the internal capabilities of the company and the degree of influence of environmental factors on it. The choice is based on the mechanism of determining the impact of the level of structural survivability on the effectiveness of projects with different innovation orientation. The results of the study on the example of petrochemical enterprises showed that in the current conditions of development, the impact on the personnel component of the production potential of the company will give the greatest effect to achieve the reliability of the development of innovative economic systems.
\end{abstract}

The modern study of world economic system development experience proves that the main factor of economical growth in modern conditions is the effective innovations. It means that the innovative technologies turn out to be the main economic resource and the effectiveness of transformations of organizational structural relations on different levels becomes the main priority in competitive surrounding.

Change of national economic direction towards innovative development is a defining factor of improvement of population's living standard, of switch to modern living standards, which as a result leads to structural changes in internal environment of economical systems as well as in integrated unions. For the last few years a lot of companies in our country have realized the necessity of continuous modernization of production and introduction of novelties, i.e. constantly working on innovative activities.

\footnotetext{
* Corresponding author: ponic7@yandex.ru
} 
Current studies of internal conditions of innovations effectiveness have led to formation of innovative liability quality as a basis to continuity of innovative activities.

However, as it was mentioned, execution of innovative projects aimed to modernize the production potential leads to emergence of perturbing reactions in the form of innovative industrial risks that have a negative impact on economic systems survivability and decrease the innovative liability.

The innovative activities in economic systems implies the transformation of innovative ideas into new or improved product, technological process introduced into production or other organizational economical changes, which reflect on the structure of the system. Therefore, the result of innovations in production-economical objects shows not only in quality of products and services and means of production, but in the combination of connections, i.e. the formation of new characteristics based on innovative changes.

In other words, innovative activity is a special purposive systematical complex of measures for using innovative potential in order to achieve a new quality of economic system after implementation of changes in the structure of production system. But changes in the structure of connections are accompanied by some negative effects for the system: structural alterations lead to growth of the system entropy due to the rise of general level of uncertainty in the changing system, destruction of new connections and emergence of new ones [1]. The emergence of this new quality leads to internal resistance of the system and to emergence of perturbing actions (innovative production risks) in case of discordance in structural transformation. As a result it may decrease the structural survivability of the system and the economic system may lose the ability to practice innovations.

Primary objective of structural changes management in this case is that these changes would lead to the increase of system's ability to function as innovative activity with positive effect along with conserving sensitivity to innovations and innovative activities in conditions of oncoming perturbing actions, i.e. to formation of innovative liability of economic system. For these purposes we analyzed the conditions of structural changes in the course of innovative activities of economic systems.

As it was said the economic system in modern conditions is a system of industrial production organization consisting of elements of production potential (staff, material and technical basis, information, finances) and combination of connections between them in terms of which the elements interact and provide unity of the system, productivity and unique character of properties under the influence of environment [2].

According to aims of innovative activity, the directions of innovative impacts in economic system differ from change of product's quality to complete upgrade of the system. It allows to highlight various levels of innovative changes: from discrete product innovations to technical organizational production upgrade. This sets the starting point of innovative alteration and the degree of potential changes in innovative liability in the process of introduction of innovations [3].

However, regardless of scales of innovative activity, its implementation influences the structure of existing system of organizational relations of economic system, causing changes of different scales, wherein the dynamics of innovative liability directly depends on the effectiveness of structural changes.

In terms of implementation of continuous innovations the quality level of structural changes plays a big part in innovative liability formation, because the influence of perturbing actions grows stronger in time.

For more detailed analysis of structural shifts caused by innovative activities in industry of the Republic Tatarstan let us consider the dynamics of these phenomena on the example of petrochemical complex as one of the defining industrial systems of the republic. The analysis data are based on the results of collaboration between the research author Krasnova A.V. and Gilyazutdinova I.V. and the official data of leading petrochemical companies of 
Tatarstan and data of Federal State Statistics Service of RT [4].

Table 1. Dynamics of innovation figures of structural shifts in petrochemical complex in RT

\begin{tabular}{|c|c|c|c|c|c|c|}
\hline Figures & \multicolumn{6}{|c|}{ Year } \\
\hline $\begin{array}{c}\text { Innovative potential } \\
\text { changes }\end{array}$ & 2002 & 2005 & 2006 & 2007 & 2008 & 2010 \\
\hline $\begin{array}{c}\text { Innovative sensitivity } \\
\text { changes }\end{array}$ & 0,02 & 0,02 & 0,2 & 0,34 & 0,21 & 0,11 \\
\hline Innovative activity changes & $\begin{array}{c}0,008 \\
2\end{array}$ & 0,008 & $\begin{array}{c}0,007 \\
8\end{array}$ & 0,077 & 0,09 & 0,013 \\
\hline
\end{tabular}

Table 1 shows that the change in innovative sensitivity caused by ongoing structural shifts gradually influences the dynamics of innovative potential development and the level of innovative activity, which means that the forming system has a vector directed to innovative development. However, during the examined period the innovative character of structural shifts in petrochemical complex is not very effective because the movement of changes in figures is not sufficient and somewhere it is slowing down or even comes to negative value. Having summed up the dynamics of data alterations from the point of innovative liability, we may come to conclusion that ongoing structural changes have a little influence on its level.

At the same time the innovation analysis of production potential development of leading organizations showed significant imbalance of innovative modernization. Further in the process of innovative activity it led to aggravation of asymmetry in production potential and increase of innovative changes level disruption of its different elements in petrochemical companies of RT and then in all the complex and led to emergence of innovative production risks of structural level.

Moreover, according to the analysis of safety management effectiveness of innovative development, its adaptability remains on the same level for last few years it terms of continuous complication of innovative processes. According to the results of research, economy in national conditions is accompanied by growth of production risks in innovative activity due to absence of balanced movements and directivity of modernization of production potential elements which led to decrease of system's structural survivability and it explains low results of innovative structural shifts and innovative activity of the complex itself.

It means that in order to achieve effective innovative development the petrochemical complex of RT needs a new management strategy based on principles of complex balanced innovated development of production potential structure of economic systems in course of its modernization. This new look on management offers formation of new model of management for structural shifts of innovative activity that will consider the level of structural survivability of economic systems choosing the innovative investment course of development. The suggested model of management has impact in two directions of structural processes quality change: at the moment of initiation (level of companies) and in the process of implementation (level of complex).

As it was mentioned before, the influence of innovative activity on economic systems of different levels is quite ambiguous in national conditions. In the course of creation of an impulse for further innovative development of economic systems the implementation of innovations is accompanied by perturbing actions. They form a source of destructive processes decreasing the innovative effect of structural transformations of the complex during cascade introduction of innovations (fig. 1) [5]. 


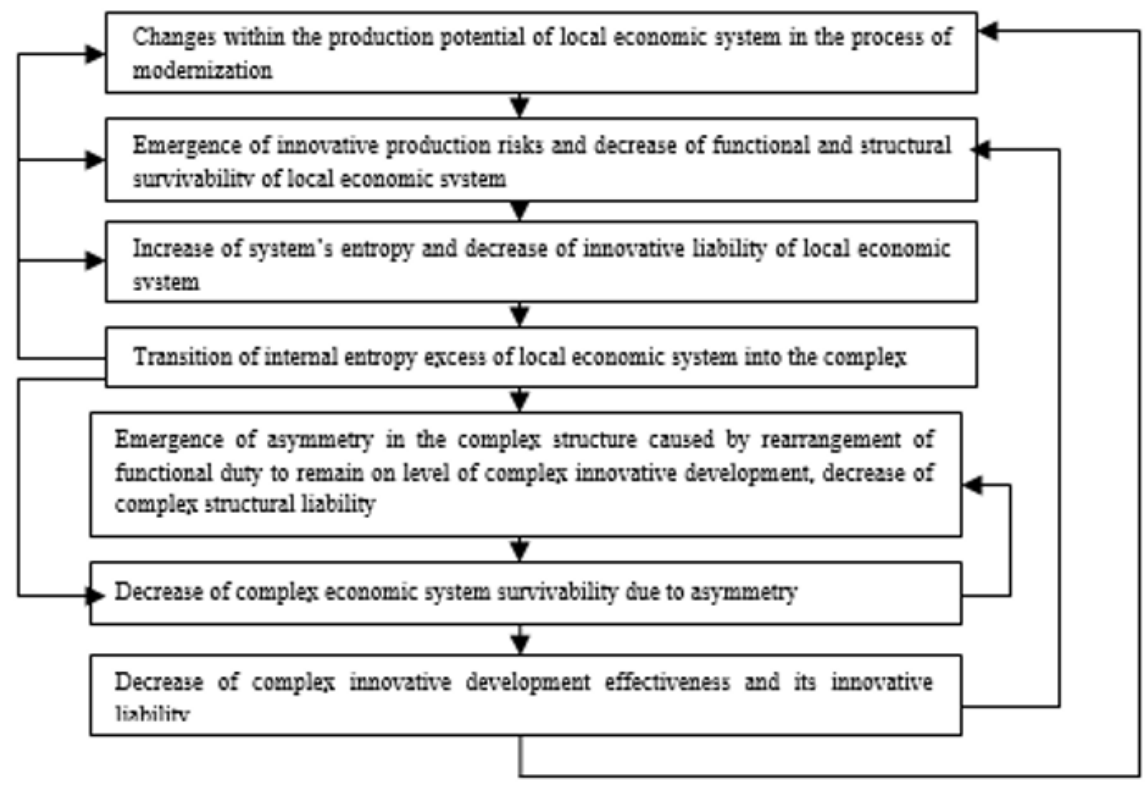

Fig. 1 Influence of local economic systems survivability on complex innovative liability in course of its structural changes

The research of local economic systems development has shown that during the innovation introduction process the elements of economic system (quality of production potential elements) are not the only ones that change. The quality characteristics of systems are also changing because systemizing connections acquire new meaning, fundamentally new forms of interaction which shows on structural changes (changes in interaction character within production potential).

At this point the relevant question is the choice of the most effective innovative investment projects within formation of management strategy for innovative structural shifts. Introduction of innovative projects, specifically in structural companies of petrochemical complex, lead to structural shifts generally in the complex.

The outer factors and resource scarcity in novelties introduction require special treatment in evaluation of projects within current management systems. Particularly package approach in analysis of investment projects, which cause structural response, allows optimizing the processes of managing structural changes and factors of innovative risks, decrease of general level of managed system entropy by the virtue of perfecting the management structure in order to increase the effectiveness of organizations innovative activity. The process of management reorganization during structure transformation of production economic activity goes within chosen management models and implements within strategies of system's innovative development.

The necessity for management system transformation is driven by requirement of adaptation of economic system in some mechanisms to a new emerging structure that often leads to delaying structure transformation process and loss of effectiveness. In terms of innovative economy the introduction of innovations accompanied by structure transformation becomes continuous process that requires new qualities from management system, among which the structural survivability becomes one of the main and it is defined by system adaptiveness. However, during the implementation of structural shifts within the management system in terms of innovative activity entropy grows by few times sometimes causing disorientation of the entire system.

That kind of phenomenon may happen if innovative development of management 
system contradicts general direction of innovative structure transformation or does not consider the speed and character of environment changes. In case of harmonious innovative development of managing and managed systems synergy of innovative process result in significant decrease of system entropy due to reduction of the emerging risks complex, level increase of structural survivability and achievement of higher results in innovative structural shift happening in observed system. That is why a relevant problem is choosing innovative investment processes based on evaluation of its innovativeness level and structural survivability of the system.

In economical literature of the last few years the central stage goes to problems of evaluation of investment instrument for impact on structural shifts of various levels. Such an analysis is required in term of uncertainty in innovative development environment and resource scarcity of industry while implementing the structural transformation. In order to do that what important is to make a complex evaluation of the observed projects from the point of safety of their introduction for economic systems with various levels of structural survivability.

The existing methods of innovative investment projects evaluation emphasize on measuring figures directly concerning the investment activity and investment processes. As a result of such evaluation findings allow determining directions and quality of investment, however, often they omit quality, complexity and timing of innovative impact on structural element of economic system in accordance with its structural survivability. That kind of management system occurs ineffective within management of safe innovative development of industrial complex.

Researches show that the classical scheme for evaluation of investments effectiveness is enough for evaluation of project effectiveness. In our opinion this approach does not give complete information on innovative structural shift and on quality of impact on structural transformation of running projects.

Another point of great importance is time of impact of investment innovative project implementation in the process of structural transformation. That is why we think it is important in the process of innovative structural shifts analysis to pay attention to determination of the moment of emergence of possibility for more effective implementation of chosen project. Such an approach will allow not only determination of the most optimal project but calculating the best time for its implementation.

Potential of the complex limits the further development in unchanging conditions. However, the implementation of optimal investment innovative project in a leading company of the complex initiates further development of structural shift which leads to formation of a new quality structure that was out of reach in old terms. This leads to efficiency growth of innovative activity, achievement of a new quality for structural survivability of economic system and increase of innovative liability of the complex accompanied by rise of innovative potential.

The basis for this evaluation approach comes from a statement that system is structurally persistent if it is able to overcome the asymmetry in rate and quality of innovative development of production potential components in the course of innovative activity. As an example of this method application let us review the evaluation of innovative projects for companies of petrochemical complex of the Republic Tatarstan (tables 2, 3) [6,8]. 
Table 2. Results of liability evaluation of innovative projects suggested for implementation in a petrochemical company of RT with various levels of survivability

\begin{tabular}{|l|l|l|l|}
\hline \multicolumn{1}{|c|}{$\begin{array}{c}\text { Level of structural } \\
\text { survivability }\end{array}$} & \multicolumn{3}{|l|}{ First innovative project (high level of innovation) } \\
\cline { 2 - 4 } & $\begin{array}{l}\text { Value of expected } \\
\text { damage before impact } \\
\text { management, rub/year }\end{array}$ & $\begin{array}{l}\text { Value of expected } \\
\text { damage after impact } \\
\text { management, rub/year }\end{array}$ & $\begin{array}{l}\text { Decrease of } \\
\text { expected } \\
\text { damage, \% }\end{array}$ \\
\hline $\begin{array}{l}\text { Low level } \\
(0-0,33)\end{array}$ & 4838941 & 4143531 & 14 \\
\hline $\begin{array}{l}\text { Medium level (0,34- } \\
0,66)\end{array}$ & 5478882 & 2972387 & 46 \\
\hline High level (0,67-1) & 8002245 & 1976671 & 75 \\
\hline
\end{tabular}

According structural survivability determination, its levels are determined on the basis of adaptiveness value of management system for innovative production risks of different value. The analysis in petrochemical companies of RT has shown that high level innovation projects are much more effective in terms of high adaptiveness of a management system for productive risks of innovative activity which allows increasing the structural survivability by virtue of this management, whereas for low adaptiveness of management system for production risks of innovative activity the effective way is to introduce perfecting innovations that do not change the production potential structure considerably.

Table 3. Results of liability evaluation of innovative projects suggested for implementation in a petrochemical company of RT with various levels of survivability

\begin{tabular}{|c|c|c|c|}
\hline \multirow{2}{*}{$\begin{array}{l}\text { Level of structural } \\
\text { survivability }\end{array}$} & \multicolumn{3}{|c|}{ Second innovative project (insignificant level of innovation) } \\
\hline & $\begin{array}{l}\text { Value of expected } \\
\text { damage before impact } \\
\text { management, rub/year }\end{array}$ & $\begin{array}{l}\text { Value of expected } \\
\text { damage after impact } \\
\text { management, } \\
\text { rub/year }\end{array}$ & $\begin{array}{l}\text { Decrease of } \\
\text { expected } \\
\text { damage, \% }\end{array}$ \\
\hline $\begin{array}{l}\text { Low level } \\
(0-0,33)\end{array}$ & 1564676 & 714686 & 54 \\
\hline $\begin{array}{ll}\text { Medium } & \text { level } \\
(0,34-0,66) & \end{array}$ & 2355910 & 1292549 & 45 \\
\hline High level $(0,67-1)$ & 3568826 & 2471296 & 31 \\
\hline
\end{tabular}

Generally, for the complex companies the most general is the medium level of management system adaptiveness, that is why it is crucial to determine the content of the innovative projects in order to reveal the component of production potential that becomes an impact on structure (fig.2).

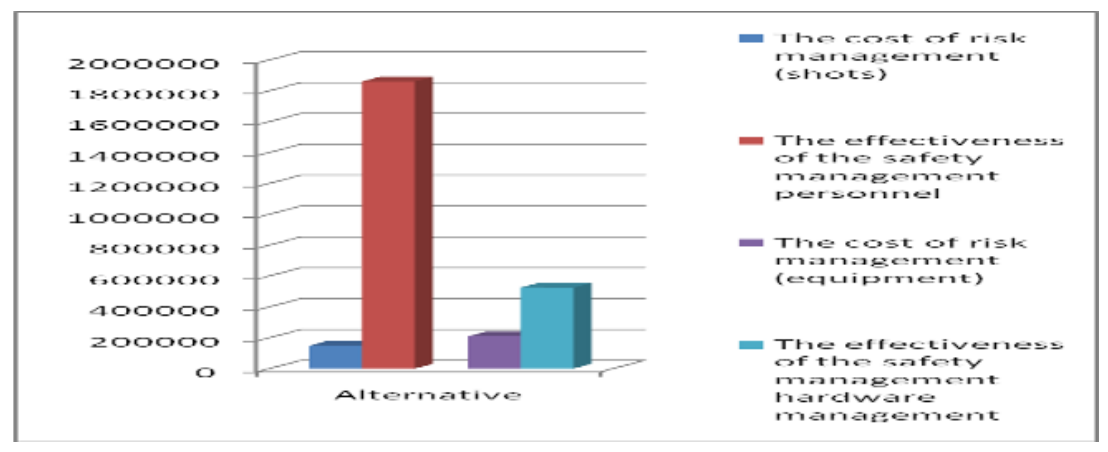

Fig. 2. Structure of the damage before and after introduction of management project for safety of personnel component (on the example of JSCo "Nizhnekamskneftekhim”)

In general, by using structural survivability value and its changes depending on 
innovative project content it is possible to determine main priority directions of innovative development giving an opportunity to increase innovative liability level of economic systems.

The management strategy for innovative structural shifts based on suggested general method will give the petrochemical complex of RT an opportunity to correct the existing model of innovative development by means of complex adaptive model of harmonious management of innovative structural shifts. The strategy implementation implies that there is some resistance to changes within subsystems of the complex, including innovations in the sphere of structural transformation which comes with the increase of the amount of risks [7]. Hence, there have to be additional mechanisms for increasing complex structural survivability within management model for innovative structural shifts in order to increase the level of its innovative liability.

Thus, in modern national conditions the management of innovative structural shifts requires the formation of management strategy which would have complex all-around impact on structural transformation in the process of innovative activity, would be targeted and able to harmonize the consequences of innovative development inconsistency in production subsystems and could be corrected in terms of changing environment conditions.

The main task of such management is to provide potential increase of economic systems innovative liability by virtue of better choice of alternatives for achieving this quality considering their structural survivability. In terms of innovative economics such a strategy demands the formation of a new unique management model for innovative structural shifts that would be advanced and match the harmonizing needs in development of separate organizations within the complex and the need for adaptiveness to environment changes and would serve as a basis for creation of a management model for economic systems innovative liability and provide the increase of its functional survivability in the process of innovation activity implementation.

\section{References}

1. L.A. Berkovich, NSU. Novosibirsk, pp. 37-47 (1985).

2.O.U. Krasilnikov, Problems of structural transformations in the economy, Economist, v. 8, pp. 52-58 (2005).

3. A.S. Ponikarova, I.R. Gilyazutdinova, Management of industrial risks of innovation at the enterprises of the petrochemical, Bulletin of Kazan Technological University, v. 4(2S), pp. 370-377 (2009).

4. I.R. Gilyazutdinova, A.S. Ponikarova, A.V. Krasnova, Some aspects of evaluating the effectiveness of management systems for innovative development of an industrial complex (on the example of the petrochemical complex of the Republic of Tatarstan), Bulletin of Kazan Technological University, v. 23, pp. 211-217 (2011).

5. A.S. Ponikarova., I.R. Gilyazutdinova, A.I. Romanova and etc. Technique of the Analysis and Assessment of Innovative Industrial Risks at Different Stages of Innovative Activity, International Journal of Economics and Financial Issues, v.6 (2S), pp.74-81 (2016).

6. A.S. Ponikarova, M.A .Zotov, E.N. Kadeeva, Balanced management of innovative industrial risks in the process of innovative development, IOP Conference Series: Materials Science and Engineering (to be published)

7. I.E. Kharlamov, S.I. Valeev, Calculation of strength of shell suffering exfoliation, Chemical and Petroleum Engineering, v.54, pp. 278-282 (2018).

8. A.D. Galeev, S.I. Ponikarov, Numerical analysis of toxic cloud generation and dispersion: A case study of ethylene oxide spill, Process Safety and Environmental Protection, v. 92, pp.702-713 (2014). 